\title{
MODELOS PARA ESTIMAR CRECIMIENTO Y EFICIENCIA DE CRECIMIENTO EN PLANTACIONES DE Pinus patula EN RESPUESTA AL ACLAREO
}

\section{MODELS FOR ESTIMATING GROWTH AND GROWTH EFFICIENCY IN Pinus patula PLANTATIONS IN RESPONSE TO THINNING}

\author{
Gerardo Rodríguez-Ortiz ${ }^{1}$, Víctor A. González-Hernández ${ }^{1 \star}$, Arnulfo Aldrete², Héctor M. De Los Santos- \\ Posadas $^{2}$, Armando Gómez-Guerrero² y Aurelio M. Fierros- González ${ }^{2}$
}

\begin{abstract}
${ }^{1}$ Postgrado en Recursos Genéticos y Productividad-Fisiología Vegetal y ${ }^{2}$ Postgrado Forestal, Colegio de Postgraduados-Campus Montecillo. Km 36.5 Carretera México -Texcoco. 56230, Montecillo, Texcoco, Edo. México. Tel. (01595) 9520200 Exts. 1584 y 1591.

*Autor para correspondencia (vagh@colpos.mx)
\end{abstract}

\section{RESUMEN}

La estrecha relación lineal entre el área foliar proyectada $(A F)$ de un árbol y el área de albura o tejido vivo del fuste $\left(A F: A_{a l b}\right)$, puede utilizarse para evaluar la eficiencia de crecimiento $(E C)$ de un bosque. El objetivo de este estudio fue estimar y analizar la relación entre el área foliar y el área de albura $\left(A F: A_{a l b}\right)$, así como la eficiencia de crecimiento en árboles de Pinus patula Schl. et Cham. plantados y sujetos a aclareos desde 2004 en Ixtlán, Oaxaca, México. En el verano de 2009 se derribaron 30 árboles en seis parcelas correspondientes a seis niveles de aclareo, en los que se estimó $A F$ y $A_{a b b}$, así como diversos indicadores de $E C$. Se encontró que el aclareo provocó ganancias significativas $(\mathrm{P} \leq 0.05)$ en $A_{a l b}, A F$, diámetros del fuste y de la rama basal, y en volumen de madera por árbol. La sección transversal del tocón $(0.30 \mathrm{~m})$ resultó la mejor sección del árbol para estimar el $A F$ con el modelo $A F=0.090\left(A_{a l b}\right)-0.395,\left(\mathrm{R}^{2}=0.82\right)$; con este modelo se estimó que cada $\mathrm{cm}^{2}$ de albura abastece a $0.082,0.090$ y $0.149 \mathrm{~m}^{2} \mathrm{de} A F$, en las secciones de tocón $(0.30 \mathrm{~m})$, a $1.30 \mathrm{~m}$ de altura e inicio de copa viva, respectivamente. El incremento medio anual en volumen (IMA $)_{v}$ se puede predecir con el modelo $I M A_{V}=3.68+0.58(A F),\left(\mathrm{R}^{2}=0.70\right)$ el cual indica que un incremento en $A F$ conduce a un aumento del volumen de madera. Si bien en este estudio la tasa anual de crecimiento en volumen de madera $\left(I M A_{V}\right)$ no se incrementó significativamente $(P \geq 0.05)$ con el aclareo $\left(12.7 \mathrm{dm}^{3}\right.$ de madera por $\mathrm{m}^{2} \mathrm{de} A F$ en parcelas con aclareo vs. 11.0 de las parcelas sin aclareo), a cinco años del aclareo el volumen total de madera por árbol en lotes con aclareo superó $(\mathrm{P}<0.05)$ de 50 a $275 \%$ al de lotes sin aclareo.

Palabras clave: Pinus patula, aclareo de árboles, albura, eficiencia del crecimiento.

\section{SUMMARY}

The strong linear relationship between projected leaf area $(L A)$ of a tree and the sapwood area $(S W)$ or living tissue in the stem (i.e., $L A: S W$ ) can be used to evaluate the growth efficiency $(G E)$ of a forest. The objective of this research was to estimate and analyze the ratio $L A: S W$ and the growth efficiency of Pinus patula Schl. et Cham. trees planted and thinned in 2004 at Ixtlán, Oaxaca, México. In the Summer 2009, 30 trees in six plots corresponding to six thinning levels, were cut in order to estimate $L A$ and $S W$ area, as well as for measuring different $G E$ indices. We found that thinning caused significant gains $(\mathrm{P} \leq 0.05)$ in $S W, A F$, stem and basal branch diameters, and in wood volume per tree. $S W$ of the transversal section of the stump $(0.30 \mathrm{~m})$ is the best tree section for estimating $L A$ with the model $L F=0.090(S W)-0.395, \quad\left(\mathrm{R}^{2}\right.$ $=0.82$ ); with this model we estimated that each square centimeter of $S W$ at the stump, at $1.30 \mathrm{~m}$ and at the living crown sections of the stem are supplying sap to $0.082,0.090$ and $0.149 \mathrm{~m}^{2}$ of $L A$, respectively. The annual increase in wood volume $\left(M A I_{V}\right)$ can be predicted as a function of $L A$ with the linear model: $M A I_{V}=3.68+0.58(L A),\left(\mathrm{R}^{2}=0.70\right)$; this model shows that wood volume $\left(M V I_{V}\right)$ would increase when $L A$ is increased. Even when in this study we found no significant effect $(P \geq$ 0.05 ) of tree thinning rates on the annual rate of wood volume growth $\left(M V I_{V}\right)\left(12.7 \mathrm{dm}^{3}\right.$ of wood per $\mathrm{m}^{2}$ of $L A$ in thinned plots $v s .11 .0$ in non thinned plot), after five years the total wood volume per tree in thinned plots was higher $(\mathrm{P}<0.05)$ by 50 to $275 \%$ compared to the non thinned control.

Index words: Pinus patula, tree thinning, sapwood, growth efficiency.

\section{INTRODUCCIÓN}

El manejo de la mayoría de masas naturales de Pinus patula Sch. et Cham. en México se hace empíricamente, sin utilizar herramientas para estimar el rendimiento esperado, como son los modelos de crecimiento. El rendimiento esperado es necesario para facilitar el proceso de toma de decisiones. En plantaciones forestales, en las que con frecuencia se incrementa la superficie plantada, tampoco se estima anticipadamente el rendimiento mediante modelos. Es conveniente que estos modelos hagan simulaciones con base en variables no destructivas de fácil medición y sensibles a las variaciones entre sitios y entre condiciones de manejo silvícola, como diámetro y altura del fuste y ramas, que además ayudarían a entender el crecimiento y el rendimiento del árbol. El aclareo es la herramienta silvícola más utilizada para el control de la densidad del rodal (parte del bosque con características homogéneas en 
especie, edad o altura) al nivel deseado (Alien et al., 2002). El aclareo aplicado en forma selectiva favorece a los árboles grandes, y es útil en rodales viejos y jóvenes (Skov et al., 2004). La intensidad del aclareo puede variar entre regiones o incluso entre sitios de una región (Brown et al., 2004). Después del aclareo los árboles remanentes (residuales) reaccionan incrementando su tasa de crecimiento (Jaakkola et al., 2005), también se hacen menos susceptibles al insecto descortezador (Dendroctonus adjunctus) y a incendios (McDowell et al., 2003), disminuyen el grado de infestación foliar causada por hongos (Kanaskie y Maguire, 2004), aumentan la asignación de biomasa al tallo (Guillespie et al., 1994), y pueden aumentar el cociente área foliar:área de albura en el tallo ( $A F: A_{\text {alb }}$ ) (Gajardo-Caviedes et al., 2005).

La eficiencia de crecimiento (EC) se define como la cantidad de madera en el fuste (volumen o biomasa) por unidad de área basal, área de albura $\left(A_{a l b}\right)$, área foliar $(A F)$ o área de copa proyectada formada por año. Para determinar los indicadores de $E C$ se requieren variables de $A F$, área foliar específica e índice de área foliar $(I A F)$, entre otras, que por estar relacionadas con la producción de madera en el fuste ayudan a entender los efectos de aclareos y otros tratamientos silvícolas sobre el crecimiento y rendimiento maderable. En ocasiones la $E C$ aumenta con el incremento en la densidad del rodal (Reid et al., 2004), pero en la mayoría de casos la $E C$ aumenta al disminuir la densidad (Curtis y Marshall, 2002). Estas discrepancias en EC se han adjudicado al método empleado en la estimación de $A F$ y a que la relación $A F: A_{\text {alb }}$ no es constante a través de diferentes densidades del rodal (McDowell et al., 2006).

El $A F$ es un factor importante en la productividad del bosque, ya que a nivel de ecosistema regula la intercepción de luz, la absorción de carbono y la transpiración (Landsberg y Gower, 1997). Sin embargo, $A F$ es difícil de medir en forma directa y por ello se utilizan ecuaciones alométricas para estimarla a nivel rama o de árbol, así como otros atributos como biomasa total, biomasa de acículas y $A F$ proyectada (Hoffmann y Usoltsev, 2002; Gilmore y Zenner, 2005). Debido a que la albura es la parte activa del xilema para el transporte de agua y nutrientes hacia las hojas, el área de dicha sección es un predictor efectivo de la tasa de transpiración y del $A F$, mediante el modelo teórico tubular (Quiñonez-Piñon y Valeo, 2004).

Pinus patula es una especie de pino endémica de México con alto potencial productivo para plantaciones de rápido crecimiento (Dvorak et al., 2000). En Ixtlán de Juárez, Oaxaca, México, el bosque natural de P. patula es el de mayor extensión (aproximadamente 5000 ha) y de mayor importancia económica (Castellanos-Bolaños et al., 2008). Por la relevancia de esta especie de pino, en Ixtlán de Juárez se estableció en 1995 una plantación de 5 ha con fines comerciales, en la cual posteriormente se aplicaron diversos niveles de aclareo. El objetivo del presente estudio fue determinar y analizar la relación área foliar:área de albura y la eficiencia de crecimiento de árboles de P. patula crecidos en esta plantación, en función del nivel de aclareo.

\section{MATERIALES Y MÉTODOS}

\section{Área de estudio}

La plantación de $P$. patula está ubicada a $12 \mathrm{~km}$ al noreste de Ixtlán, Oaxaca, a $17^{\circ} 22^{\prime} 38.69^{\prime \prime}$ LN y $96^{\circ} 28^{\prime} 44.8^{\prime \prime}$ LO, a $2550 \mathrm{~m}$ de altitud. La empresa "Comunal, Forestal y Agropecuaria (UCFAS)", propietaria de la plantación, la plantó en 1995 en 5 ha de montaña cuyos terrenos variaban en pendiente, desde terrenos casi planos hasta con $40 \%$ de pendiente, después de preparar el suelo con maquinaria pesada. La plántula utilizada se obtuvo de semilla colectada de 50 árboles seleccionados del bosque natural de la misma comunidad. En 2004 la empresa delimitó seis parcelas de 20 x $20 \mathrm{~m}$, separadas entre sí por una franja de protección de 10 a $15 \mathrm{~m}$ de ancho. En cinco parcelas aplicó tres aclareos de diversas intensidades, en los años 2004, 2006 y 2008, y dejó una parcela intacta como testigo, como se detalla en el Cuadro 1. Estas seis parcelas constituyeron los seis tratamientos evaluados en el presente estudio. Al momento de realizar cada aclareo los técnicos de la empresa midieron las siguientes variables en cada árbol: diámetro del tallo a $1.30 \mathrm{~m}(\mathrm{DN}, \mathrm{cm})$, altura total del tallo (AT, $\mathrm{m})$, área basal del tallo $\left(\mathrm{AB}, \mathrm{m}^{2}\right)$ y volumen por árbol y de aclareo $\left(\mathrm{m}^{3}\right)$.

Para el manejo experimental, en este estudio iniciado en 2009 todos los valores medidos y calculados se extrapolaron a nivel parcela y hectárea, y se calculó el incremento medio anual en volumen por árbol y hectárea con la fórmula: $I M A_{V}=$ volumen/edad de plantación (Will et al., 2002) para el periodo 1995-2009, con base en los datos de inventario de la empresa.

\section{Muestreo de acículas y madera}

Con datos obtenidos en 2009 se generó la estructura diamétrica (distribución de frecuencias por categoría del diámetro a $1.30 \mathrm{~m}$ ) y se caracterizó el nivel de dominancia de copa de cada parcela. Para medir el área foliar y la biomasa por árbol, la empresa permitió derribar 30 árboles representativos de la plantación (muestreo destructivo). Para ello, en forma aleatoria se seleccionaron cinco árboles por parcela, sanos y sin bifurcación; de ellos, tres se clasificaron en campo como suprimidos, siete intermedios, 15 codominantes y cinco dominantes. El derribo se realizó con una motosierra Husqvarna ${ }^{\circledR}$ modelo 2009 (Estocolmo, Suecia) a una altura de corte de $30 \mathrm{~cm}$ sobre el suelo. 
Cuadro 1. Porcentaje global extraído de la densidad inicial de plantación y por año de aclareo, densidad de árboles e indicadores de follaje en parcelas aclareadas de $P$. patula en Ixtlán, Oaxaca.

\begin{tabular}{|c|c|c|c|c|c|c|c|c|c|}
\hline \multirow[b]{2}{*}{ Parc. } & \multirow[b]{2}{*}{ Aclareo $(\%)^{\dagger}$} & \multicolumn{3}{|c|}{$\begin{array}{l}\text { Densidad extraída por aclareo } \\
\qquad(\%)^{\dagger \dagger}\end{array}$} & \multicolumn{2}{|c|}{$\begin{array}{l}\text { Densidad } \\
(\text { árboles ha-1) }\end{array}$} & \multicolumn{2}{|c|}{ IF 2009} & \multirow{2}{*}{$\begin{array}{l}\text { Volumen } \\
\text { ( } \mathrm{dm}^{3} / \text { árbol) }\end{array}$} \\
\hline & & 2004 & 2006 & 2008 & Inicial & Residual & $\mathrm{AF}$ (m²/árbol) & $\begin{array}{l}\text { Biomasa foliar } \\
\text { (kg/árbol) }\end{array}$ & \\
\hline 1 & 65.3 & 30.9 & 9.3 & 25.1 & 3600 & 1250 & 24.2 & 5.0 & 220 \\
\hline 3 & 68.7 & 49.2 & 1.6 & 17.9 & 5675 & 1775 & 20.4 & 4.0 & 160 \\
\hline 4 & 64.0 & 41.3 & sa & 22.7 & 5000 & 1800 & 10.5 & 1.9 & 160 \\
\hline 5 & 58.0 & sa & $\mathrm{sa}$ & 58.0 & 4525 & 1900 & 13.6 & 2.8 & 150 \\
\hline 2 & 39.7 & 15.1 & sa & 24.6 & 3650 & 2200 & 13.6 & 2.9 & 120 \\
\hline 6 (test.) & 22.0 & sa & $\mathrm{sa}$ & $\mathrm{sa}$ & 4650 & $3625^{\circ}$ & 8.2 & 1.6 & 80 \\
\hline Promedio & & & & & 4517 & 2092 & 15.1 & 3.0 & 150 \\
\hline
\end{tabular}

${ }^{\dagger}$ Porcentaje de densidad global cortada con respecto a la densidad inicial de plantación. ${ }^{\dagger+}$ Porcentaje de densidad cortada con respecto a la densidad inicial por año de intervención. 'Reducción por autoaclareo. sa = sin aclareo; Parc = parcela; AF = área foliar; IF = indicadores de follaje.

Las variables medidas en los árboles derribados fueron: diámetro de tocón con corteza (DT, en cm), diámetro normal (DN) y diámetro al inicio de copa viva (DCV); altura del tocón $(\mathrm{cm})$, a la punta (AT), de fuste limpio (AFL) y de copa viva $(\mathrm{ACV})$. En cada árbol derribado fueron separadas las ramas y fuste, y en cada rama se registró: posición en la copa (dividida en tres secciones: inferior, media y superior), altura de inserción ( $\mathrm{AR}$, en $\mathrm{m}$ ), diámetro basal a $1 \mathrm{~cm}$ del corte (DBR, en $\mathrm{cm}$ ) con ayuda de un vernier marca Scala $222 \mathrm{~A} \AA$. El fuste completo se fraccionó en trozas de $1 \mathrm{~m}$ de largo, y en cada troza se midió diámetro inferior y superior, y el árbol se cubicó por el método de trozas sobrepuestas (Bailey, 1995).

Para el muestreo de acículas se seleccionó aleatoriamente una submuestra de 20 árboles obtenidos de todas las parcelas (cuatro árboles de las parcelas 1 y testigo, y tres para el resto). La copa de cada árbol elegido se dividió en tres secciones de igual longitud, y en cada sección se eligieron al azar de dos a tres ramas de cada uno de los 20 árboles (145 ramas en total), para con ellas representar toda la longitud del fuste. Las acículas separadas de las ramas se transportaron al laboratorio en bolsas de papel debidamente identificadas, para su medición. En los puntos de medición de diámetro (DT, DN y DCV) y en la parte media en el fuste, se obtuvieron rodajas de $5 \mathrm{~cm}$ de espesor aproximadamente, que se identificaron y guardaron en costales de yute; este material también se llevó a laboratorio para medir el volumen de cada rodaja, y su biomasa después de secado en estufa por $72 \mathrm{~h}$ a $102^{\circ} \mathrm{C}$.

Con estos datos se calculó la densidad específica de la madera de cada rodaja y del promedio por árbol, promedio que según Deene y Hale (1999) es confiable. La densidad estimada fue luego multiplicada por el volumen del árbol para así estimar la biomasa $\left(B_{\text {árbol }}, \mathrm{kg}\right)$. Las hojas frescas muestreadas fueron escaneadas en escáner de cama plana HP Scanjet G4050® para obtener una imagen de su área proyectada. El $A F\left(\mathrm{~cm}^{2}\right)$ por imagen se obtuvo mediante el programa Image ${ }^{\circledR}$ y los valores sumados generaron el $A F$ por rama. Para obtener el $A F$ por árbol $\left(A F_{\text {árbol }}\right)$ se ajustó el modelo polinomial de regresión a nivel rama que se muestra en resultados y conclusiones. Las acículas se secaron a $75^{\circ} \mathrm{C}$ por $72 \mathrm{~h}$ para medir la biomasa foliar por rama $\left(B_{\text {foliar }}, \mathrm{g}\right)$. En cada rodaja se midió: diámetros sin corteza, de albura y de duramen (diferenciadas por el método translúcido) y con aplicación de azul de bromofenol $1 \%$, que en $24 \mathrm{~h}$ colorea de azul el duramen y de amarillo la albura (Gilmore et al., 1996); y ancho y área de albura ( $A_{a l b}$, al asumir circularidad concéntrica) mediante la siguiente expresión (Turner et al., 2000):

$$
\left.A_{\text {alb }}=\left\{\left[\pi\left(\frac{D_{s c}}{2}\right)^{2}\right]-\left[\pi\left(\frac{D_{s c}}{2}\right)-A_{\text {ancho }}\right)^{2}\right]\right\}
$$

donde $A_{a l b}$ es el área de albura en la rodaja de cada sección $\left(\mathrm{cm}^{2}\right) ; D_{s c}$ el diámetro sin corteza de la rodaja $(\mathrm{cm}) ; \pi=$ 3.1416; y $A_{\text {ancho }}$ el ancho promedio de albura $(\mathrm{cm})$, medido en cuatro ejes.

El efecto del aclareo sobre el $A F$ proyectada y sobre el área de albura en el fuste, se evaluó para cada sección del fuste mediante el modelo logarítmico $A F=\beta_{0}\left(A_{a l b}\right)^{\beta 1}$ propuesto por Will et al. (2002), con el método SUR (seemingly unrelated regressions) de SAS (SAS Institute, 2004), en el que $\mathrm{AF}=$ área foliar por árbol $\left(\mathrm{m}^{2}\right), \mathrm{A}_{\mathrm{alb}}=$ área de albura $\left(\mathrm{cm}^{2}\right)$, y $\beta_{0}, \beta_{1}=$ parámetros de regresión a estimar. Este efecto se calculó para el periodo de 5 años transcurridos desde el primer aclareo (Cuadro 1). 


\section{Análisis estadísticos}

La $A F_{\text {árbol }}$ se relacionó mediante regresión con el $A_{\text {alb }}$ de cada sección del fuste (tocón, DN y DCV), con modelos de regresión lineal simple. En los datos se comprobaron los supuestos de normalidad y homogeneidad de varianzas con el procedimiento UNIVARIATE de SAS (SAS Institute, 2004). También se usaron los procedimientos GLM, MODEL, TTEST (comparación de medias entre los árboles bajo aclareo y testigo) y CORR de SAS.

\section{RESULTADOS Y DISCUSIÓN}

\section{Relación área foliar: área de albura $\left(A F: A_{a l b}\right)$}

El AF $\left(\mathrm{cm}^{2}\right)$ de ramas individuales $\left(A F_{\text {rama }}\right)$ se estimó mediante el ajuste de la siguiente ecuación lineal con la muestra de 145 ramas, al considerar que el área de conducción total está afectada por la posición en la copa:

$$
A F_{\text {rama }}=475.80+534.86(A B R)+147.61(A B R) X_{1}+242.12(A B R) X_{2}
$$

donde $A F_{\text {rama }}$ es el área foliar por rama $\left(\mathrm{m}^{2}\right), A B R$ es el área basal de rama $(\pi / 4 \times$ cuadrado del diámetro basal de rama, en $\mathrm{cm}^{2}$ ), y $X_{1,2,3}$ es 1 si la rama estaba en la sección 1 (copa inferior), 2 (copa media) o 3 (copa superior). Con una bondad de ajuste $\mathrm{R}^{2}=0.87$ y un $\mathrm{CV}=38 \%$, el modelo estimó $2806 \mathrm{~cm}^{2}$ de $A F$ promedio por rama, con un error estándar de $89 \mathrm{~cm}^{2}$. Hubo diferencias entre las tres secciones del fuste en cuanto a los parámetros de sus modelos para estimar la relación $A F: A_{a l b}$, que en los tres casos fueron ecuaciones de tipo lineal. En las secciones de inicio de copa viva y de diámetro de tocón los modelos $A F\left(m^{2}\right)=0.082\left(A_{\text {alb }}\right)-0.665$ y $A F=0.149\left(A_{\text {alb }}\right)-0.173$, respectivamente, tuvieron iguales bondades de ajuste $\left(\mathrm{R}^{2}\right.$ $=0.85)$, coeficientes de variación $(\mathrm{CV}=22 \%)$ y errores estándar (0.60), ligeramente mejores que en la sección del tocón $\left(\mathrm{R}^{2}=0.82, \mathrm{CV}=24 \%\right.$ y error estándar $\left.=0.66\right)$, cuya ecuación lineal fue $A F\left(m^{2}\right)=0.090\left(A_{a l b}\right)-0.395$. La Figura 1 ilustra el comportamiento de datos observados y predichos de $A F$ para el caso de la sección del tocón.

Estos resultados difieren de los encontrados por GajardoCaviedes et al. (2005) en Nothofagus dombeyi, quienes con un modelo potencial $\left(y=\beta_{0} e^{\beta_{1} x}\right)$ estimaron el $A F$ proyectada en función de la $A_{a l b}$. Debido a que en $P$. patula las ecuaciones obtenidas en las tres secciones del fuste no mostraron diferencias $(\mathrm{P} \geq 0.63)$ en el intercepto $\beta_{0}$, se ajustaron sin éste, lo que además facilitó su comparación con las reportadas por otros autores. Con el modelo $\sin \beta_{0}$ se estimó que cada unidad de área de albura $\left(\mathrm{cm}^{2}\right)$ abastece 790, 880 y $1480 \mathrm{~cm}^{2}$ de $A F$, para las secciones de tocón, $\mathrm{DN}$ e inicio de copa viva, respectivamente. Estos valores son inferiores a los encontrados por Stancioiu y O'Hara (2005) en árboles de 28 a 80 años de Sequoia sempervirens
(D. Don) Endl., en los que se reportaron relaciones de 2500, 4000 y $5700 \mathrm{~cm}^{2} \mathrm{~cm}^{-2}$ para DT, DN y DCV, respectivamente. Las discrepancias en el valor del cociente $A F: A_{\text {alb }}$ pueden ser debidas a variaciones entre especies y en la edad de los árboles, que en este estudio eran jóvenes (14 años), ya que según Stancioiu y O'Hara (2005) dicho cociente tiende a aumentar con la edad del rodal.

El modelo de área foliar $\left(A F=0.114 \times A_{\text {alb }}^{1.01}\right)$ permitió detectar que en un periodo de 5 años (tiempo transcurrido del primer aclareo al año de muestreo - Cuadro 1), únicamente en la sección de inicio de copa viva hubo efecto significativo ( $\mathrm{P} \leq 0.04)$ del aclareo en $A F$ proyectada, cuya bondad de ajuste $\left(\mathrm{R}^{2}=0.85\right)$ fue más alta y su error estándar (0.58) fue menor que en las demás secciones.

En los árboles de $P$. patula usados en este estudio, el $A_{\text {alb }}$ de los individuos en parcelas aclareadas fue superior (110.7 $\left.\mathrm{cm}^{2}\right)$ que los de la parcela testigo sin aclarear $\left(60.2 \mathrm{~cm}^{2}\right)(\mathrm{P} \leq$ $0.05)$, promedios que se estimaron mediante la ecuación de regresión ajustada para ese punto, con $A F_{\text {árbol }}$ de 16.3 y 8.8 $\mathrm{m}^{2}$ para las parcelas aclareadas y el testigo, respectivamente; la prueba de t confirmó las diferencias $(\mathrm{P} \leq 0.04)$ entre los árboles aclareados y los del testigo. En rodales de 75 años de $P$. ponderosa Laws, Simonin et al. (2006) encontraron que la porosidad del área de albura fue mayor en árboles sujetos a aclareos que en rodales no intervenidos en un tiempo de respuesta de 6 años, lo que implica que el aclareo incrementó la proporción de tejido conductivo en albura. Una situación similar podría haber sucedido en los árboles de $P$. patula aquí estudiados.

Estudios previos en diversas especies forestales han indicado que la producción adicional de follaje debida al aclareo podría ser explicada por el incremento en la disponibilidad de nutrientes, agua y luz, lo que a su vez permite generar un centro de copa más bajo debido a que hay mayor supervivencia de las ramas inferiores y mayor crecimiento de las ramas laterales (Baldwin et al., 2000; Yu et al., 2003). Este comportamiento se observó en $P$. patula, una especie intolerante a la sombra y de difícil poda natural (Dvorak et al., 2000), ya que el diámetro basal de rama fue superior en parcelas con aclareo que sin aclareo (1.56 vs. $1.16 \mathrm{~cm}$, respectivamente; $\mathrm{P} \leq 0.04$ ), y las parcelas $1 \mathrm{y}$ 3 con la menor densidad residual dieron los valores más altos (Cuadro 1). Según Eckmüllner y Sterba (2000), la relación $A F: A_{\text {alb }}$ puede variar por diferencias asociadas con el diámetro de vasos conductores en el xilema, amplitud de anillos o velocidad del crecimiento. En este sentido, si se supone que el crecimiento rápido puede generar mayor conductividad hidráulica al generar haces vasculares de mayor diámetro, explicaría el que los árboles de $P$. patula de parcelas aclareadas hayan tenido una mayor relación $A F: A_{\text {alb. }}$ 


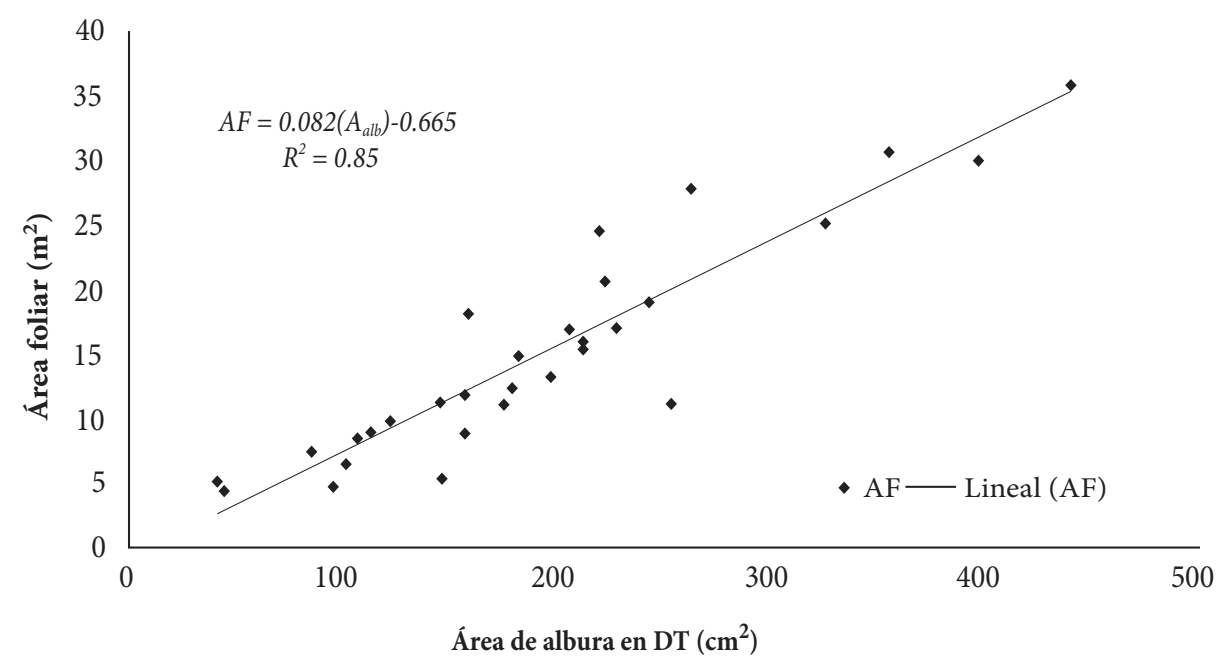

Figura 1. Relación lineal entre el área foliar $(\mathrm{AF})$ y el área de albura $\left(\mathrm{A}_{\mathrm{alb}}\right)$ en la sección de diámetro de tocón en árboles de P. patula.

\section{Eficiencia de crecimiento $(E C)$}

La $E C$ fue determinada mediante índices de crecimiento que relacionan el volumen o biomasa de madera con área de albura o área foliar. En cuanto al cociente volumen de madera en el fuste:área de albura en la sección de DN, el aclareo más alto $(68.7 \%)$ de la parcela 3 dio un valor de $1.08 \mathrm{dm}^{3} \mathrm{~cm}^{-2}$, y así esta parcela fue superior $(\mathrm{P} \leq 0.01)$ que el nivel de aclareo más bajo (39.7\%, parcela 2) que sólo produjo $0.92 \mathrm{dm}^{3} \mathrm{~cm}^{-2}$. El índice de crecimiento basado en la biomasa de madera en el fuste $\left(B_{\text {mad }}\right)$ por unidad de área de albura $\left(A_{\text {alb }}\right)$ en la sección transversal de $\mathrm{DN}$, fue más alto $(\mathrm{P} \leq 0.03)$ en la parcela 3 (de mayor intensidad de aclareo) con $0.86 \mathrm{~kg} \mathrm{~cm}^{-2}$, que en la parcela 1 (aclareo de $30.9 \%$ en 2004, Cuadro 1) con $0.6 \mathrm{~kg} \mathrm{~cm}^{-2}$.

El índice de crecimiento obtenido con el cociente volumen de madera en el fuste:AF, mostró su valor más alto con el aclareo de $64.0 \%$ (parcela 4 ) donde se produjo $17.8 \mathrm{dm}^{3}$ de madera por $\mathrm{m}^{2}$ de $A F$. Los valores más bajos se encontraron en las parcelas 1 y 2 (con 65.3 y $39.7 \%$ de aclareo, respectivamente), ambos inferiores a la parcela 4 ( $\mathrm{P} \leq 0.04$ y $\mathrm{P} \leq 0.05$, respectivamente) (Figura 2a); el valor mínimo fue de $9.6 \mathrm{dm}^{3} \mathrm{~m}^{-2}$ y ocurrió en el nivel de aclareo más bajo (parcela 2). La biomasa de madera por unidad de $A F$ mostró una tendencia similar, con el valor más alto $\left(13.15 \mathrm{~kg} \mathrm{~m}^{-2}\right.$ ) en el aclareo de $64.0 \%$ (parcela 4), que superó al nivel más bajo $(39.7 \%)$ de aclareo ( $\leq$ $0.04)$, que sólo produjo $6.82 \mathrm{~kg}$ de biomasa por unidad de $A F$ (Figura 2b). En todos los índices determinados las parcelas con más alta eficiencia de crecimiento fueron las de mayor intensidad de aclareo, sobre todo cuando éste fue intenso desde el principio (2004). Las parcelas con las mínimas intensidades de aclareo en 2004 (parcelas 1 y 2 con
30.9 y $15.1 \%$, respectivamente; Cuadro 1), tuvieron una EC incluso más baja $(\mathrm{P} \leq 0.05)$ que el testigo (Figura 3 ).

Muchos investigadores han observado una disminución en la $E C$ conforme aumenta el tamaño del árbol o del $A F$ (Ryan et al., 1997; McDowell et al., 2002; Will et al., 2002; Mainwaring y Maguire, 2004). Esta misma situación se detectó en el aclareo de $65.3 \%$ donde están los árboles de mayor volumen o biomasa maderable y mayor $A F$, aunque su eficiencia de crecimiento fue la más baja, incluso menor que en el testigo (parcela 6). Este comportamiento es similar al encontrado por McDowell et al. (2003) en $P$. ponderosa. Este resultado podría deberse a que las parcelas de menor densidad residual (parcelas 1 y 2) tenían los árboles más grandes después de las cortas, y posiblemente estos árboles hayan destinado una menor proporción de carbono asimilado para la producción de madera en el fuste y una mayor proporción de carbono para formar las raíces estructurales y éstas pudieran mantener la estabilidad en los espacios que se abrieron con los aclareos McDowell et al., 2003).

Otro aspecto que podría estar asociado con los mecanismos de reducción de la EC conforme aumenta el tamaño del árbol, es la conductividad hidráulica la cual se reduce conforme crece el árbol, según lo encontrado por Magnani et al. (2000) y McDowell et al. (2002). Las parcelas con aclareo tuvieron en promedio $160 \mathrm{dm}^{3} /$ árbol, el doble que el testigo con $80 \mathrm{dm}^{3} /$ árbol (Cuadro 1), como resultado de la elevada densidad residual (3625 árboles ha ${ }^{-1}$ ) de este último. Esto pudo haberse originado por la competencia asimétrica, que según Schwinning y Weiner (1998) y Binkley et al. (2004) sucede cuando hay competencia severa y sólo un pequeño número 

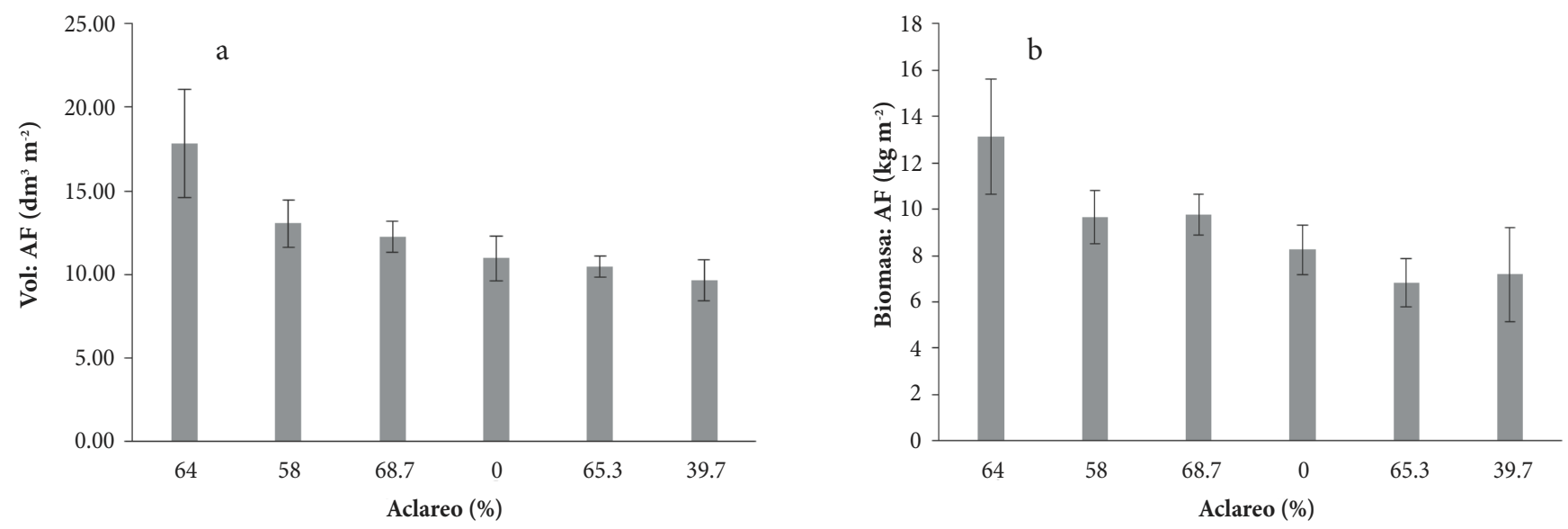

Figura 2. Volumen (a) y biomasa (b) de madera producida por unidad de área foliar ( $A F$ ) en función del la intensidad del aclareo (árboles cortados de la densidad inicial de plantación) aplicado a Pinus patula. Las barras verticales representan el error estándar.

de árboles grandes (dominantes) obtienen la mayor parte de los recursos disponibles y los utilizan eficientemente, mientras que los árboles suprimidos sólo pueden usar los recursos que no aprovechan los árboles que dominan un sitio. Esta diferencia se nota con mayor claridad al comparar los volúmenes por árbol de la parcela testigo $\left(80 \mathrm{dm}^{3} /\right.$ árbol $)$ y la parcela 1 ( $220 \mathrm{dm}^{3} /$ árbol; Cuadro 1$)$, este último con la menor densidad residual (1250 árboles ha $\left.{ }^{-1}\right)$, mayor tiempo de respuesta al aclareo y mayor cantidad de biomasa foliar por individuo (5 kg/árbol).

El efecto de aumentar el volumen de aclareo en el crecimiento del árbol depende del tiempo de respuesta. Los mayores volúmenes de aclareo en 2004 (parcelas 3 y 4) y 2008 (parcela 5) tuvieron EC más altas que el testigo, y aquellos con los menores volúmenes cortados en 2004 mostraron EC más bajas que el testigo (parcelas 1 y 2 )

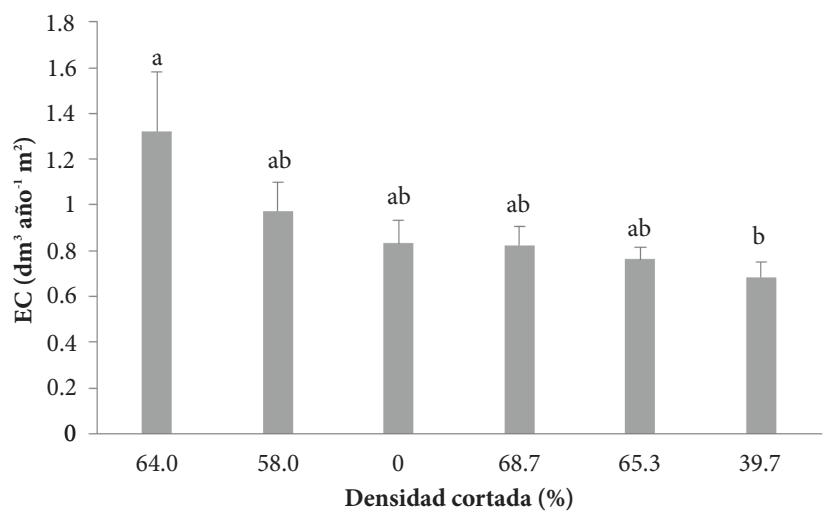

Figura 3. Comparación de medias de eficiencia del crecimiento (EC) en función de la intensidad del aclareo (medida en porcentaje de árboles cortados con respecto a la densidad inicial de plantación) aplicado a $P$. patula. Las líneas verticales representan el error estándar; letras iguales corresponden a media iguales (Tukey, $0.03 ; \mathrm{CV}=33 \%$; E.E. $=0.29$ ).
(Cuadro 1, Figura 2). Según McDowell et al. (2003), en una plantación con fase de descanso tiende a existir una mayor proporción de carbono aéreo y subterráneo. Es posible que esto haya ocurrido en los árboles de este estudio, ya que los suelos de la plantación se encontraban en su fase de descanso después de un uso agrícola, con la consecuente alta proporción de carbono por árbol en el suelo; a ello también se atribuye la respuesta poco clara de la época de aclareo en el crecimiento del árbol.

Otro índice que determina la $E C$ está basado en el incremento medio anual en volumen $\left(I M A_{V}\right)$ y el $A F$. La parcela 4 con intensidad de aclareo de $64.0 \%$ tuvo la $E C$ más alta, pues cada $\mathrm{m}^{2}$ de $A F$ formó $1.32 \mathrm{dm}^{3}$ de madera por año, valor que supera a la parcela $2(\mathrm{P} \leq 0.03)$ la cual tuvo menor intensidad de aclareo y presentó la producción más baja de madera con $0.68 \mathrm{dm}^{3}$ año ${ }^{-1} \mathrm{~m}^{-2}$ de $A F$ (Figura 3). El hecho que el testigo (cuya densidad cortada fue cero) haya mostrado una $E C$ más alta que las parcelas con aclareo, es una respuesta típica según McDowell et al. (2007), ya que el sitio ocupa su máxima capacidad de soporte a través del autoaclareo.

El $I M A_{V}$ se ajustó mediante un modelo lineal que utiliza $A F$ como variable independiente (Figura 4). El coeficiente de ajuste del modelo fue $\mathrm{R}^{2} 0.70$, y los dos parámetros del modelo fueron significativos $(\mathrm{P} \leq 0.005)$. Debido que la pendiente $\left(\beta_{1}\right)$ de la ecuación fue 0.58 , ello significa que cada incremento unitario de AF genera un incremento de $0.58 \mathrm{dm}^{3}$ de madera por año; así, la $E C$ disminuyó con el aumento de $A F_{\text {arbol }}$ como lo evidencia la correlación negativa entre ambas $(r=-0.4)$. Este comportamiento del $A F$ está probablemente regulado por el incremento en la disponibilidad de recursos asociado con una menor competencia residual, ya que en experimentos controlados de $P$. ponderosa, Naumburg et al. (2001) encontraron una 


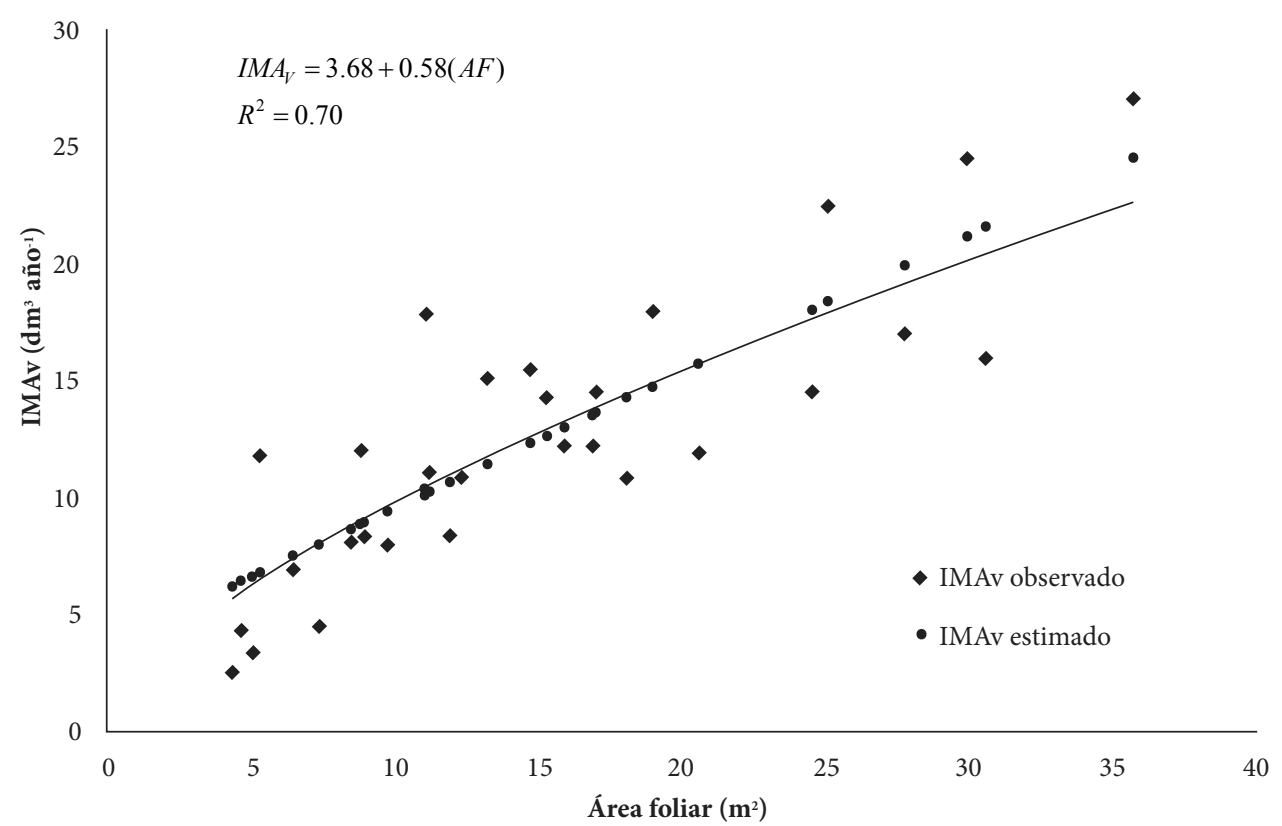

Figura 4. Incremento medio anual en volumen $\left(I M A_{v}\right)$ en función de área foliar $(A F)$ en árboles de Pinus patula en Ixtlán de Juárez, Oaxaca.

reducción en la tasa de fotosíntesis neta y en la biomasa de toda la planta en respuesta a la sombra. Además, el incremento de agua, nutrientes y otros recursos podría también ser crítico en la respuesta del $A F$ (McDowell et al., 2007). El $A F$ por unidad de superficie disminuyó al reducirse la densidad luego de ser aplicados los aclareos, porque se redujo la proporción de copas por los espacios abiertos. No obstante, después del aclareo los árboles tienden a crecer a una tasa mayor y así dan lugar a mayores valores de $A F$ y de volumen de madera por árbol. Este comportamiento se observó en este estudio, donde el AF y el volumen mostraron una correlación positiva $(\mathrm{r}=0.85 ; \mathrm{P} \leq$ $0.03)$, lo mismo que la biomasa foliar y volumen de madera $(\mathrm{r}=0.83 ; \mathrm{P} \leq 0.03)$, en concordancia con lo encontrado por McDowell et al. (2006) en P. ponderosa.

\section{CONCLUSIONES}

El área de albura de la sección transversal del tocón se puede usar para estimar el área foliar proyectada y la eficiencia del crecimiento en bosques de Pinus patula sujetos a aclareo. En Ixtlán de Juárez, Oaxaca, cada unidad de albura $\left(\mathrm{cm}^{2}\right)$ en las secciones del tocón, diámetro normal e inicio de copa viva, abastece a 0.082, 0.090 y $0.149 \mathrm{~m}^{2}$ de área foliar proyectada, respectivamente. El incremento medio anual en volumen en esta especie se puede predecir con el modelo lineal $I M A_{V}=3.68+0.58(A F)^{5}$, cuya cinética muestra que el aumento en área foliar $(A F)$ genera una ganancia en el incremento medio anual de volumen en madera $\left(I M A_{v}\right)$ y la eficiencia de crecimiento alcanza un valor máximo de $1.32 \mathrm{dm}^{3} \mathrm{año}^{-1} \mathrm{~m}^{-2}$.

Con respecto al testigo, el aclareo de árboles indujo incrementos significativos en la proporción de tejido conductivo en albura, en el área foliar por árbol, en el diámetro de la rama basal y en el volumen de madera por árbol. Además se encontró que el diámetro del tocón se puede usar para detectar diferencias en el efecto del aclareo sobre el área foliar. Sin embargo, la eficiencia del crecimiento, medida como incremento medio anual en volumen por unidad de área foliar, no se incrementó significativamente con el aclareo, pues a los 5 años las parcelas aclareadas produjeron $12.7 \mathrm{dm}^{3}$ de madera por metro cuadrado de área foliar proyectada $v s .11 \mathrm{dm}^{3} \mathrm{~m}^{-2} \mathrm{del}$ testigo no aclareado.

\section{BIBLIOGRAFÍA}

Alien C D, Savage, D A Falk, K F Sucklin, T W Swetnam, T Schulke, P B Stacey, P Morgan, M Huffman, J T Klingel (2002) Ecological restoration of southwestern ponderosa pine ecosystems, a broad perspective. Ecol. Appl. 12:1418-1433.

Bailey R L (1995) Upper-stem volumes from stem-analysis data: an overlapping bolt method. Can. J.For. Res. 25:170-173.

Baldwin V C, K D Peterson, A Clark, R B Ferguson, M R Strub, D R Bower (2000) The effects of spacing and thinning on stand and tree characteristics of 3-year-old loblolly pine. For. Ecol. Manage. 137:91-102.

Binkley D, J L Stape, M G Ryan (2004) Thinking about efficiency of resource use in forests. For. Ecol. Manage. 193:5-16. 
Brown R T, J K Agée, J F Franklin (2004) Forest restoration and fire: principles in the context of place. Conserv. Biol. 18:903-912.

Castellanos-Bolaños J F, E J Treviño-Garza, O A Aguirre-Calderón, J Jiménez-Pérez, M Musalem-Santiago R López-Aguillón (2008) Estructura de bosques de pino pátula bajo manejo en Ixtlán de Juárez, Oaxaca, México. Madera y Bosques 14:51-63.

Curtis R O, D D Marshall (2002) Level-of-growing-stock cooperative study in Douglas-fir. Rep. 14. Stampede Creek: 30 Year Results. USDA For. Serv. Res. Pap. PNW-RP-543. USA. 120 p.

Deene M, M Hale (1999). Cell wall and lumen percentages in relation to wood density of Nothofagus nervosa. IAWA Journal 20:23-36.

Dvorak W S, G R Hodge, J E Kietzka, F Malan, L F Osorio T K Stanger (2000) Pinus patula. In: Conservation and Testing of Tropical and Subtropical Forest Tree Species by the CAMCORE Cooperative, College of Natural Resources, NCSU. Raleigh, NC. USA. pp:148173.

Eckmüllner O, H Sterba (2000) Crown condition, needle mass, and sapwood area relationships of Norway spruce (Picea abies). Canadian Journal of Forest Research 30:1646-1654.

Gajardo-Caviedes P A, M A Espinoza, U T González, D G Ríos (2005) The influence of thinning and tree size on the sapwood area/leaf area ratio in coigue. Can. J. For. Res. 35:1679-1686.

Gilmore D W, E K Zenner (2005) Foliage-sapwood area relationships for balsam fir in Northwestern central Minnesota. Northern J. Appl. For. 22:203-210.

Gilmore D W, R S Seymour, D A Maguire (1996) Foliage-sapwood area relationships for Abies balsamea in central Maine, USA. Can. J. For. Res. 26:2071-2079.

Guillespie A, H L Alen, J Vose (1994) Amount and vertical distribution of foliage of young loblolly pine as affected by canopy position and silvicultural treatment. Can. J. For. Res. 24:1337-1344.

Hoffmann C W, V A Usoltsev (2002) Tree-crown biomass estimation in forest species of the Ural and of Kazakhstan. For. Ecol. Manage. 158:59-69.

Jaakkola T, H Mäkinen, M P Saren, P Saranpää (2005) Does thinning intensity affect the tracheid dimensions of Norway spruce? Can. J. For. Res. 35:2685-2698.

Kanaskie A, D Maguire (2004) Trends in Swiss needle cast damage in thinned and unthinned Douglas-fir plantations with varying intensity of Swiss needle cast in the Coast Range of Oregon. In: 2004 Annual Report. Swiss Needle Cast Cooperative, College of Forestry, Oregon State University, Corvallis. Ore. USA. pp:17-23.

Landsberg J J, S T Gower (1997) Applications of Physiological Ecology to Forest Production. Academic Press. San Diego, Calif. USA. 354 p.

Magnani F, M Mencuccini, J Grace (2000) Age-related decline in stand productivity: the role of structural acclimation under hydraulic constraints. Plant Cell Environ. 23:251-264.

Mainwaring D B, D A Maguire (2004) The effect of local stand structure on growth and growth efficiency in heterogeneous stands of ponderosa pine and lodgepole pine in central Oregon. Can. J. For.
Res. 34:2217-2229.

McDowell N G, H Barnard, B J Bond, T Hinckley, R Hubbard, H Ishii, B Kostner, F C Meinzer, J D Marshall, F Magnani, N Phillips, M G Ryan, D Whitehead (2002) The relationship between tree height and leaf area:sapwood area ratio. Oecologia 132:12-20.

McDowell N G, H D Adams, J D Bailey, M Hess, T E KoIb (2006) Homeostatic maintenance of ponderosa pine gas exchange in response to stand density changes. Ecol. Appl. 16:1164-1182.

McDowell N G, H D Adams, J D Bailey, T E Kolb (2007) The role of stand density on growth efficiency, leaf area index, and resin flow in southwestern ponderosa pine forests. Can. J. For. Res. 37:343-356.

McDowell N G, J R Brooks, S Fitzgerald, B J Bond (2003) Carbon isotope discrimination and growth response of old ponderosa pine trees to stand density reductions. Plant Cell Environ. 26:631-644.

Naumburg E, L E DeWaId, T E KoIb (2001) Shade responses of five grasses native to southwestern U.S. Pinus ponderosa forests. Can. J. Bot. 79:1001-1009.

Quiñonez-Piñon R, C Valeo (2004) Sapwood area as a scaling factor for transpiration rates. In: American Geophysical Union, Spring Meeting 2004. Montreal, Canada. 17-21 May, 2004. pp:1818-1851.

Reid D E, V J lieffers, U Silins (2004) Growth and crown efficiency of height repressed lodgepole pine; are suppressed trees more efficient? Trees (Berl.) 18:390-398.

Ryan M G, D Binkley, J H Fownes (1997) Age-related decline in forest productivity: pattern and process. Adv. Ecol. Res. 27:213-262.

SAS Institute Inc (2004) SAS/STAT 9.1 User's guide. SAS Institute, Cary, NC. USA. 4979 p.

Schwinning S, J Weiner (1998) Mechanisms determining the degree of size asymmetry in competition among plants. Oecologia 113:447455.

Simonin K, T E KoIb, M Montes-Helu, G W Koch (2006) Restoration thinning and the influence of tree size and leaf area to sapwood area ratio on Pinus ponderosa Laws water relations. Tree Physiol. 26:493-503

Skov K R, T E KoIb, K F Wallin (2004) Tree size and drought affect ponderosa pine physiological response to thinning and burning treatments. For. Sci. 50:81-91.

Stancioiu P T, K L O'Hara (2005) Sapwood area-leaf area relationships for coast redwood. Can. J. For. Res. 35:1250-1256.

Turner D P, S A Acker, J E Means, S L Garman (2000) Assessing alternative allometric algorithms fro estimating leaf area of Douglas-fir trees and stands. For. Ecol. Manage. 126:61-76.

Will R E, G T Munger, Y J Zhang, B E Borders (2002) Effects of annual fertilization and complete competition control on current annual increment, foliar development, and growth efficiency of different aged Pinus taeda stands. Can. J. For. Res. 32:1728-1740.

Yu S, J L Chambers, Z Tang, J P Barnett (2003) Crown characteristics of juvenile loblolly pine 6 years after application of thinning and fertilization. For. Ecol. Manage. 180:345-352. 